\title{
Designing Networks with Low Structural Congestion via Game Theory and Linear Programming
}

\author{
${ }^{1}$ Ahmad Askarian and ${ }^{2}$ Andras Farago \\ Department of Electrical Engineering and Computer Science, University of Texas at Dallas, \\ United States; \\ 1ahmad.askarian@utdallas.edu; ${ }^{2}$ farago@utdallas.edu
}

\begin{abstract}
We propose a network topology design approach that targets the reduction of structural congestion in a directed acyclic network. What we mean by structural congestion is that a node has much higher in-degree than out-degree in a directed network. We approach the issue using a network design game model. In this model we consider multiple sources and one destination. Each node is willing to connect to other nodes but it should pay the price of whole paths it uses to send traffic to the destination. The model yields a weight for each link. We show that if these weights are used to compute shortest paths, then a network topology is obtained with a low level of structural congestion.

The proposed method has two phases. In Phase I, we solve a linear optimization problem in order to find the optimum link weights. In Phase II, each node optimizes its own individual objective function, which is based on the weights computed in Phase I. We show that there exists a Nash Equilibrium which is also the global optimum. In order to measure the penalty incurred by the selfish behavior of nodes, we use the concept called price of anarchy. Our results show that the price of anarchy is zero.
\end{abstract}

Keywords - Communication network; Game theory; Linear programming

\section{Introduction}

Communication network design methods and algorithms are approached with various types of design goals. Minimum vulnerability, fault tolerance and quality of services are often used in this context [1].

As network nodes become more intelligent, distributed algorithms become increasingly dominant. Although a centralized algorithm which optimizes the entire network configuration would maximize efficiency and utilization, it is not as stable as distributed algorithm. Stability in a network means that if some nodes fail, other nodes have the capability to reconfigure themselves and recover from the failure. This idea can lead to a decision making algorithm that is executed in each node separately to optimize the global benefit.

One step further in this direction is when a node does not know the global benefit or does not care about it. In this situation a network involves selfish agents, making decisions to optimize their own benefit [2]. Social and biological networks are examples of such selfishly behaving agents that form a network. Game theory is a useful tool to analyze and predict the behavior of this kind of networks. 
In this work, we study a directed acyclic network design game in the light of structural congestion consideration. Each node in a network which has a high in-degree is a bottleneck. It is desirable to avoid such a structural bottleneck, as it can easily lead to traffic congestion.

Our main objective here is to show that there exists a well-defined utility function in which the selfish behavior of each node leads to a network topology with minimum structural congestion. To do that first we convert a minimum structural congestion problem into a shortest path routing problem, in which link weights are obtained as the output of a linear optimization task. Then we construct a utility function in order to encourage each node to use paths with minimum overlap. The path set forms a new network which has a minimal structural congestion.

The rest of this paper is organized as follows. After discussing related work in section II, we define the concept of structural congestion and optimization framework for analyzing network topology in section III. In section IV we derive a condition in which selfish behavior of each node can lead to an optimum. Finally, conclusion is presented in section V.

\section{Related Work}

The design of various networks have been studied in sociology, natural sciences and engineering for a long time [3]. Optimization and graph theory was the most useful tool in this field, since Myerson introduced a new network design model using game theory for social and economic networks [4]. After that, the concept of game theoretic models have been used in different communication networking contexts, such as routing [5], flow control [6] and dynamic access control in wireless networks [7].

Nash Equilibrium has been considered as a way to quantify the performance associated with selfish behavior of each player. Such equilibria are inefficient [12]. The lack of global control can lead to suboptimal network performance. The "price of anarchy" is a concept in game theory which measures the inefficiency of a system due to selfish behavior of each player [13].

A comprehensive study of game theory based communication network design is [1], which involves three important design considerations, namely the price of establishing a link, path delay, and path proneness to congestion. They showed that there exists an equilibrium point which is a global optimum.

The cost function which they considered in [1] for each player in a network design game considering path congestion is:

$$
C\left(v_{i}\right)=\max _{v_{k} \in V} \max _{v_{j} \in l\left(v_{i}, v_{k}\right)} \eta_{G}^{\text {in }}\left(v_{k}\right)
$$

In which $\eta_{G}^{\text {in }}\left(v_{k}\right)$ is the input degree of a node $v_{k}$ in a graph $G$, and $l\left(v_{i}, v_{k}\right)$ denotes the path connecting $V_{i}$ and $V_{k}$. In this method each node is required to connect to all other nodes and they show that a directed ring is both an optimum and equilibrium.

In this study, we focus on the structural congestion of the network. For our purposes, the network can be represented by a weighted directed acyclic graph.

\section{Structural Congestion}

A path in a network is a sequence of links, each link (except the first) having the same start node as the end node of the previous link in the sequence. Each link has a utilization factor, which we call 
Link Utilization (LU). If we view the network topology as a set of paths from a source to a destination, it contains several links which have different LU. A path's proneness to congestion is depending on the maximum LU on the path from a source to a destination. Let us look at a node $V_{i}$ in the network, which is described using the graph $G(N, E)$. Let $\eta_{i}^{\text {in }}$ and $\eta_{i}^{\text {out }}$ be the input and output degree of $V_{i}$. We define the Degree Ratio (DR) for each node as follow:

Definition: The degree ratio of a node $i \in N$ is $D R_{i}=\frac{\eta_{i}^{\text {in }}}{\eta_{i}^{\text {out }}}$.

Assuming all links have unit capacity, the quantity $D R_{i}$ shows the structural congestion at the node. High $D R_{i}$ means node $i \in N$ is a bottleneck and can be a point of congestion. There is a direct relationship between $D R_{i}$ in a network and the Maximum Link utilization (MLU) which is described in the following conjecture.

Conjecture: A set of paths in a directed acyclic network which minimizes MLU, will form a new network which minimizes (at least approximately) $D R_{i}$ for all $i \in N$ and carries the same amount of traffic.

Minimizing MLU means finding a set of paths between a source and a destination, such that these paths split the input traffic as much as possible and, at the same time, have a minimum overlap. First we analyze the problem of minimizing MLU, because it is a linear optimization problem. Consider a directed acyclic graph $G(N, E)$ which represents the network. $C_{i j}:(i, j) \in E$ is a set of edge capacities and $\left(s_{k}, t_{k}\right)$ is a set of source-destination pairs for each session $k \in K$. The percentage of traffic on a link $(i, j) \in E$ that belongs to session $k$ is $X_{i j}^{k}$. With these notations, the formulation is [8]:

$$
\begin{aligned}
& \min _{X} M L U \\
& \sum_{j:(i, j) \in E} X_{i j}^{k}-\sum_{j:(j, i) \in E} X_{j i}^{k}=\left\{\begin{array}{cc}
1 & i \varepsilon S \\
-1 & i \varepsilon T \\
0 & \text { otherwise }
\end{array}\right. \\
& \sum_{k \in K} X_{i j}^{k} \leq C_{i j} \cdot M L U \\
& X_{i j} \geq 0 \in \varepsilon
\end{aligned}
$$

Using duality theory we can write the dual optimization problem as follows:

$$
\begin{aligned}
& \max _{P, W} \sum_{k \in K} p_{t_{k}}^{k} \\
& \sum_{(i, j) \in E} C_{i j} w_{i j}=1 \\
& p_{i}^{k}-p_{j}^{k} \leq w_{i j} \\
& p_{s_{k}}^{k} \\
& w_{i j} \geq 0
\end{aligned}
$$

Because the primal and dual problems are both linear, strong duality holds and according to complementary slackness in the KKT theorem if $\hat{X}_{i j}^{k}$ is an optimal solution for the primal problem, and $\left\{\hat{w}_{i j}, \hat{p}_{i j}^{k}\right\}$ is an optimal solution for the dual, then we have: 


$$
\hat{X}_{i j}^{k} \cdot\left(\hat{p}_{i}^{k}-\hat{p}_{j}^{k}+\hat{w}_{i j}\right)=0
$$

This equation indicates that if sessionk uses link $(i, j) \in E$ then $p_{j}^{k}-p_{i}^{k}=w_{i j}$. According to the Duality Theorem, if $\left\{\hat{w}_{i j}\right\}_{(i, j) \in E}$ is used as link metric in a shortest path algorithm, all non-empty links $\left(X_{i j}^{k}>0\right)$ will be selected in a shortest path algorithm procedure [8]. As a result if any shortest path algorithm uses $\left\{\hat{w}_{i j}\right\}_{(i, j) \in E}$ as link weights we will have set of paths between a source and destination which has an important character. The path set splits the input traffic as much as possible through the network and at the same time has minimum number of overlap links.

A network topology with minimum structural congestion means that $D R_{i}$ is close to one. Let us consider a weighted directed acyclic graph which represents a network with only one sourcedestination pair and the capacity of all links are 1 . Weights are calculated on the basis of the dual optimization problem discussed above. If we run any shortest path algorithm over such a network we obtain a set of paths $\Omega$. If we delete any link $(i, j) \in E$ which is not on a member of $\Omega$ we will have a new weighted acyclic graph which represents a new network. All nodes in the new network have an equal or smaller $D R_{i}$ than the old one.

\section{Network Design Model}

In this section we study the performance of a non-cooperative network. This means, each node (player) tries to maximize its own benefit. The network design goal is minimizing the structural congestion. Node $V_{i}$ gains $\alpha_{i}$ by connecting to any node in the network. So each node tries to make a connection to as many nodes as possible. By connecting to each node it must calculate the length of a path from itself to a destination. The gain a node can achieve by connecting to others minus the summed length of all paths heading to destination form the utility function of each node as follows.

Node Utilization:

$$
u_{G}\left(v_{i}\right)=\alpha_{i}\left|S_{i}\right|-\sum_{p \in P} d_{G(W)}^{p}\left(v_{i}, v_{t}\right)
$$

Which $\left|S_{i}\right|$ is the number of output links in node $V_{i}$ and $d_{G(W)}^{p}\left(v_{i}, v_{t}\right)$ is a distance between node $V_{i}$ and the destination using path $p \in P$ in the designed network using links weight $W$.

The network utilization is the sum of all node utilization functions.

Network Utilization:

$$
U_{G}=\sum_{i \in N} u_{G}\left(v_{i}\right)
$$

Optimum solution for such a game happens when we have a maximum $U_{G}=\sum_{i \in N} u_{G}\left(v_{i}\right)$. But in order to find equilibrium point we need to analyze the selfish behavior of each node. For that purpose consider Figure 1 as a part of a network. Node $V_{i}$ is deciding to stay on its current strategy 
(connection to other nodes) or deviate (drop a connection or make a new one) based on the maximum utilization function.

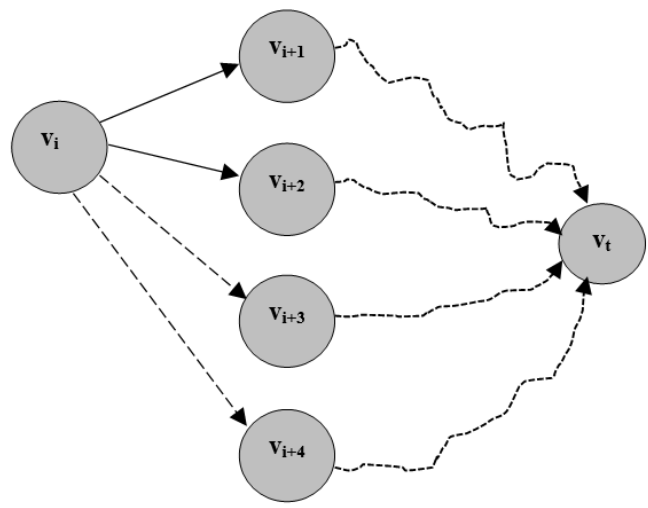

Figure 1: Node $\mathbf{V}_{\mathrm{i}}$ decision strategies

Consider node $V_{i}$ in the network. It is already connected to nodes $V_{i+1}$ and $V_{i+2}$. It should decide to connect to nodes $V_{i+3}$ and $V_{i+4}$ or not. The current topology is represented by the graph $G_{1}$, if it connect to $V_{i+2}$ the graph will be $G_{2}$ and if it connect to both $V_{i+1}$ and $V_{i+2}$ the graph will be $G_{3}$. Based on the weight system in the network the distance from nodes $v_{i+1}, v_{i+2}, v_{i+3}$ and $V_{i+4}$ to destination are $l_{i+1}, l_{i+2}, l_{i+3}$ and $l_{i+4}$ respectively. The utility of node $v_{i}$ is:

$$
\begin{gathered}
u_{G_{1}}\left(v_{i}\right)=2 \alpha_{i}-\left(w_{i, i+1}+l_{i+1}+w_{i, i+2}+l_{i+2}\right) \\
u_{G 2}\left(v_{i}\right)=3 \alpha_{i}-\left(w_{i, i+1}+l_{i+1}+w_{i, i+2}+l_{i+2}+w_{i, i+3}+l_{i+3}\right) \\
u_{G 3}\left(v_{i}\right)=4 \alpha_{i}-\left(w_{i, i+1}+l_{i+1}+w_{i, i+2}+l_{i+2}+w_{i, i+3}+l_{i+3}\right. \\
\left.+w_{i, i+4}+l_{i+4}\right)
\end{gathered}
$$

Suppose that based on the weight system, links $(i, i+1),(i, i+2)$ and $(i, i+3)$ are on the shortest paths. So we have:

$$
l_{S h-P}^{i}=w_{i, i+1}+l_{i+1}=w_{i, i+2}+l_{i+2}=w_{i, i+3}+l_{i+3}
$$

If we want that the selfish behavior of the node $v_{i}$ leads to optimum topology, then the following conditions must hold:

$$
\begin{aligned}
& u_{G_{1}}\left(v_{i}\right)<u_{G 2}\left(v_{i}\right) \\
& u_{G 2}\left(v_{i}\right)>u_{G 3}\left(v_{i}\right)
\end{aligned}
$$

So we have:

$$
w_{i, i+1}+l_{i+1}<\alpha_{i}<w_{i, i+4}+l_{i+4}
$$

If we consider $\lambda_{S h-P}^{i}$ as the length of a second shortest path from the node $v_{i}$ to the destination we have: 


$$
l_{S h-P}^{i}<\alpha_{i}<\lambda_{S h-P}^{i}
$$

This is the condition in which selfish behavior of each node in the network will lead to optimum topology with minimum structural congestion. Now the question is if there is any upper and lower bound for $\alpha$ in general. Using topological sorting theorem [9] we can find such a bound. Based on topological sorting theorem a directed acyclic graph can be represented in way that nodes index increase when they get closer to the destination and there is no link $(m, n)$ if $m>n$. For example a directed acyclic graph with 4 nodes after topological sorting is shown in figure 2.

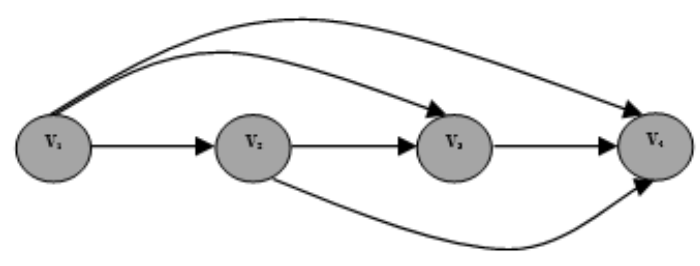

Figure 2: Topological Sorting

After topological sorting we suppose that node 1 is the source and node $\mathrm{N}$ is the destination. Now it is clear that after using weight set which is the solution of dual optimization problem in section III we have $l_{S h-P}^{i}>l_{S h-P}^{i+1}$. So the lower bound for $\alpha$ is $l_{S h-P}^{1}$ which is the shortest path from source to the destination. Also we have $\lambda_{S h-P}^{i}>\lambda_{S h-P}^{i+1}$. So the upper bound for $\alpha$ is:

$$
\lambda_{S h-P}^{N-2}=\max \left\{w_{N-2, N-1}+w_{N-1, N}, w_{N-2, N}\right\}
$$

So we have:

$$
l_{S h-P}^{1}<\alpha<\lambda_{S h-P}^{N-2}
$$

Now consider the network in figure 2. The question is what is the upper and lower bound for $\alpha$ in this network. Table I shows the optimal weights which calculate using dual optimization problem in section III.

\section{Table 1: Optimum Weights}

\begin{tabular}{|l|l|}
\hline$p_{1}-p_{2}=w_{12}$ & 1 \\
\hline$p_{1}-p_{3}=w_{13}$ & 1 \\
\hline$p_{1}-p_{4}=w_{14}$ & 2 \\
\hline$p_{2}-p_{3}=w_{23}$ & 3 \\
\hline$p_{2}-p_{4}=w_{24}$ & 1 \\
\hline$p_{3}-p_{4}=w_{34}$ & 1 \\
\hline
\end{tabular}

In this case upper and lower bound is:

$$
\begin{aligned}
& l_{S h-P}^{1}=2 \\
& \lambda_{S h-P}^{N-2}=4
\end{aligned}
$$


So $\alpha=3$ satisfies the condition. After applying $\alpha=3$ in the node utility function, node $v_{2}$ can improve its utility function by deviate from current strategy to the one which has no connection to node $V_{3}$. As a result we have network with better structural congestion. Applying this method to all nodes the result would be a network topology with minimum structural congestion.

In order to analyze the price of selfish behavior there is two important concepts which are price of stability and price of anarchy. The price of stability is the ratio between best objective function value in equilibrium point and the optimum network utilization function. On the other hand price of anarchy is the ratio between worse objective function value in the equilibrium and the optimum network utilization function [10]. In this section we showed that price of stability is one and anarchy is free if each node applies the node utilization function. Otherwise price of anarchy is depend on $\alpha$ and $\left\{w_{i j}\right\}_{(i, j) \in E}[1]$.

Figure 3 shows how the optimization method provides inputs for our network design game.

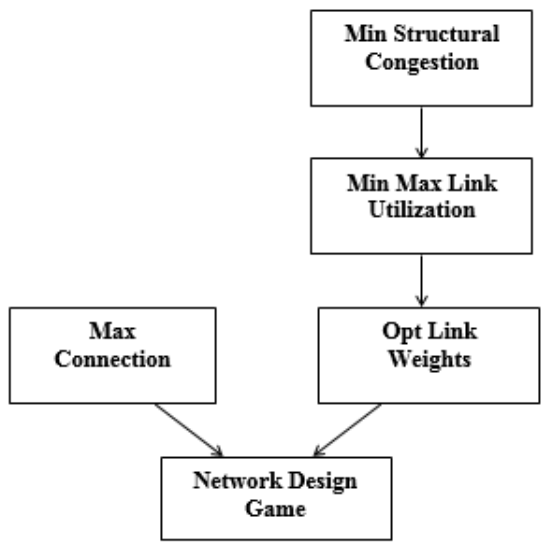

Figure 3: Algorithm Flowchart

It is worth mentioning that the described method can be implemented in a network using distributed algorithms like the Bellman-Ford Algorithm [11]. It means that it is not necessary for each node to have information about the whole network. It is only needed to know the parameter $\alpha$, the weights of its outgoing links and the distance of its neighbors to the destination. Having this information is sufficient to find an optimum strategy.

\section{Simulation}

For the simulation we consider a directed acyclic network with 20 nodes. All links have a capacity one and we consider node 1, 2 and 3 as a source of traffic and node 20 as the destination. Figure 4 shows the network topology. Maximum degree ratio is 19 in this network. Each node minimizes its own objective function based on optimum link weights and its desire to make more connection. 


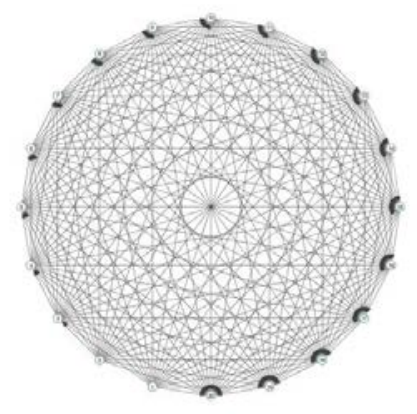

Figure 4: Network Topology

After solving the dual optimization problem we have $l_{S h-P}^{1}=4.3$ and $\lambda_{S h-P}^{18}=14.5$. Figure 4 shows that no structural congestion is a result of choosing $4.3<\alpha<14.5$, it means that $D R_{i}=1$ for all $i \in N$. As $\alpha$ deviates from the constraint each node is more willing to make a new connection and it leads to more structural congestion. For example if we choose $\alpha=20$ degree ratio of nodes 16 and 17 are high and they can be considered as a network bottleneck.

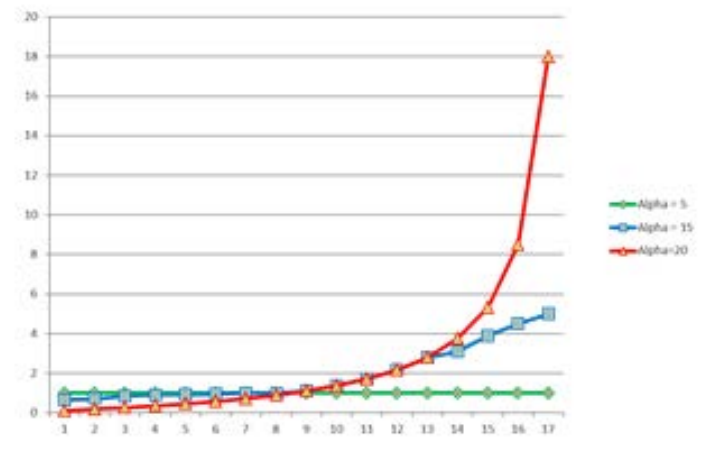

Figure 5: Degree ratio for each node using different alpha

\section{Conclusion}

This paper investigates the question "how non-cooperative nodes in a network can create an efficient network?" We have studied the result of the selfish behavior of nodes, and compares it to the situation in which there is a central control unit in the network. Central control can force all nodes to use a predefined strategy in which the network utilization is optimum.

Based on the discussion in section IV if we fix the benefit of establishing a new link for each node, $\alpha$, in a way that satisfies the condition $l_{S h-P}^{1}<\alpha<\lambda_{S h-P}^{N-2}$, the price of stability will be one and also the price of anarchy will be zero in this network design game.

\section{REFERENCES}

[1] Nahir, A., et al. Topology Design of Communication Networks: A Game-Theoretic Perspective. IEEE/ACM Trans. Netw. 22(2): (2014) 405-414.

[2] Page Jr, F. H., et al. Strategic basins of attraction, the path dominance core, and network formation games. Games and Economic Behavior 66.1 (2009): 462-487. 
[3] Jackson, M., et al. On the formation of interaction networks in social coordination games. Games and Economic Behavior 41.2 (2002): 265-291.

[4] Aumann, R., et al. Endogenous formation of links between players and coalitions: an application of the Shapley value. The Shapley Value (1988): 175-191.

[5] Fotakis, D., et al. The structure and complexity of Nash equilibria for a selfish routing game. Theoretical Computer Science 410.36 (2009): 3305-3326.

[6] Fang, Z., et al. Fair bandwidth sharing algorithms based on game theory frameworks for wireless ad-hoc networks. INFOCOM 2004. Twenty-third AnnualJoint Conference of the IEEE Computer and Communications Societies. Vol. 2. IEEE, 2004.

[7] Ji, Z., et al. Cognitive radios for dynamic spectrum access-dynamic spectrum sharing: A game theoretical overview. Communications Magazine, IEEE 45.5 (2007): 88-94.

[8] Wang, Y., et al. Internet traffic engineering without full mesh overlaying. INFOCOM 2001. Twentieth Annual Joint Conference of the IEEE Computer and Communications Societies. Proceedings. IEEE. Vol. 1. IEEE, 2001.

[9] Varol, Y., et al. An algorithm to generate all topological sorting arrangements. The Computer Journal 24.1 (1981): 83-84.

[10] Roughgarden, T. Selfish routing and the price of anarchy. Vol. 174. Cambridge: MIT press, 2005.

[11] Awerbuch, B., et al. Approximate distributed bellman-ford algorithms. Communications, IEEE Transactions on 42.8 (1994): 2515-2517.

[12] G. Debreu, A social equilibrium existence theorem, Proc. Nat. Acad. Sci., vol. 38, pp. 886-893, Oct. 1952.

[13] Koutsoupias, E., et al. Worst-case equilibria. STACS 99. Springer Berlin Heidelberg, 1999. 